03;04;09

\title{
Новый метод подавления периферического поглощения в лазерно-плазменном источнике коротковолнового излучения с Хе газоструйной мишенью
}

\author{
(С) П.С. Буторин ${ }^{1}$, С.Г. Калмыков ${ }^{2, \uparrow}$, М.Э. Сасин ${ }^{2}$ \\ ${ }^{1}$ Санкт-Петербургский политехнический университет Петра Великого, \\ Санкт-Петербург, Россия \\ ${ }^{2}$ Физико-технический институт им. А.Ф. Иоффе РАН, Санкт-Петербург, \\ Россия \\ E-mail: Serguei.Kalmykov@mail.ioffe.ru
}

Поступило в Редакцию 19 апреля 2018 г.

Экспериментально наблюдалось более чем десятикратное увеличение интенсивности EUV-излучения из лазерно-плазменного источника при освещении $\mathrm{Xе}$ газоструйной мишени широким лазерным лучом, охватывающим всю ее наиболее плотную часть, по сравнению с интенсивностью излучения при традиционной геометрии, когда луч остро сфокусирован на ось струи. В качестве объяснения предполагается, что в этом случае почти вся плотная часть струи под действием лазерного облучения нагревается, подвергается ионизации и становится прозрачной для квантов исследуемого излучения. Толщина же поглощающей периферической оболочки лазерной искры соответственно уменьшается, что приводит к увеличению интенсивности доходящего до наблюдателя излучения.

DOI: 10.21883/PJTF.2018.23.47018.17340

В течение нескольких последних лет продолжаются усилия по внедрению EUV-литографии (EUV - extreme ultraviolet) в промышленность. Обсуждается два варианта этой технологии: с излучением на длине волны $\lambda=13.5 \mathrm{~nm}$ от лазерно-плазменного источника с мишенью из твердого $\mathrm{Sn}$ либо на длине волны $\lambda=11.2 \mathrm{~nm} \mathrm{c} \mathrm{,чистым“}$ источником, в котором используется газовая Хе мишень $[1,2]$. В последнем случае излучателем является облачко плотной Хе плазмы с 
размером порядка $100 \mu \mathrm{m}$. Спектр такой плазмы представляет собой континуальный пик без дискретных линий в полосе $\lambda \approx 10-15 \mathrm{~nm}$ [3-6], что позволяет использовать различные длины волн, но перспектива вывода EUV-литографии с Хе источником на промышленный уровень критическим образом зависит от интенсивности излучения в указанной полосе.

Обычно исследования лазерно-плазменного источника с Хе мишенью выполняются при острой фокусировке (с диаметром фокального пятна в десятки микрометров) лазерного луча на самую плотную часть газоструйной мишени вблизи ее оси. Такая конфигурация оптимальна для лазерного пробоя и последующего нагрева плазмы, но при этом излучающее в EUV-диапазоне плазменное ядро окружено периферической оболочкой из нейтрального и относительно низкоионизованного Хе, фотоионизационное поглощение EUV-излучения в котором на обеих упомянутых длинах волн превышает $90 \%$. На это обстоятельство обращалось внимание еще в самых ранних исследованиях [7-9]. Предпринимались попытки [7-13] уменьшить это поглощение, однако достигнутый выигрыш в выходе EUV-излучения оказался невелик (не более чем в 2.5 раза). В настоящей работе рассматривается новый оригинальный метод подавления периферического поглощения.

Схема эксперимента показана на рис. 1. Лазерная искра возбуждалась в истекающей из сопла Лаваля сверхзвуковой микроструе Хе сфокусированным лучом $\mathrm{Nd}$ :YAG-лазера $(\lambda=1.064 \mu \mathrm{m})$ с длительностью импульса $\tau_{\text {las }} \approx 10 \mathrm{~ns}$ и доводимой до плазмы энергией $E_{\text {las }} \approx 0.93 \mathrm{~J}$. Обсуждаемая далее геометрия облучения струи определялась характеристиками объектива: выходная апертура луча составляла $28 \mathrm{~mm}$, задний фокальный отрезок $-70 \mathrm{~mm}$. Сопло установлено на трехкоординатном позиционирующем устройстве, позволяющем перемещать его в пространстве с точностью до $5 \mu \mathrm{m}$ по отношению к неподвижной точке фокуса. Расстояние от оси лазерного луча до плоскости выходного отверстия сопла в описываемых экспериментах составляло $\Delta X=1 \mathrm{~mm}$. Газовые условия в струе-мишени рассчитывались с помощью гидродинамического моделирования [12,13]. Полученный в [13] расчетный радиальный профиль плотности газа приведен на рис. 2. Видно, что для условий эксперимента плотность Хе на оси струи составляла $n_{0}=7 \cdot 10^{18} \mathrm{~cm}^{-3}$, а ширина профиля на его полувысоте $\Delta_{0.5}=0.7 \mathrm{~mm}$. EUV-излучение плазмы отражалось от интерференционного зеркала $\mathrm{Si} / \mathrm{Mo} \mathrm{c} \mathrm{эффективной} \mathrm{полосой} \mathrm{отражения} \lambda=13.5 \pm 0.3 \mathrm{~nm}$, проходило 


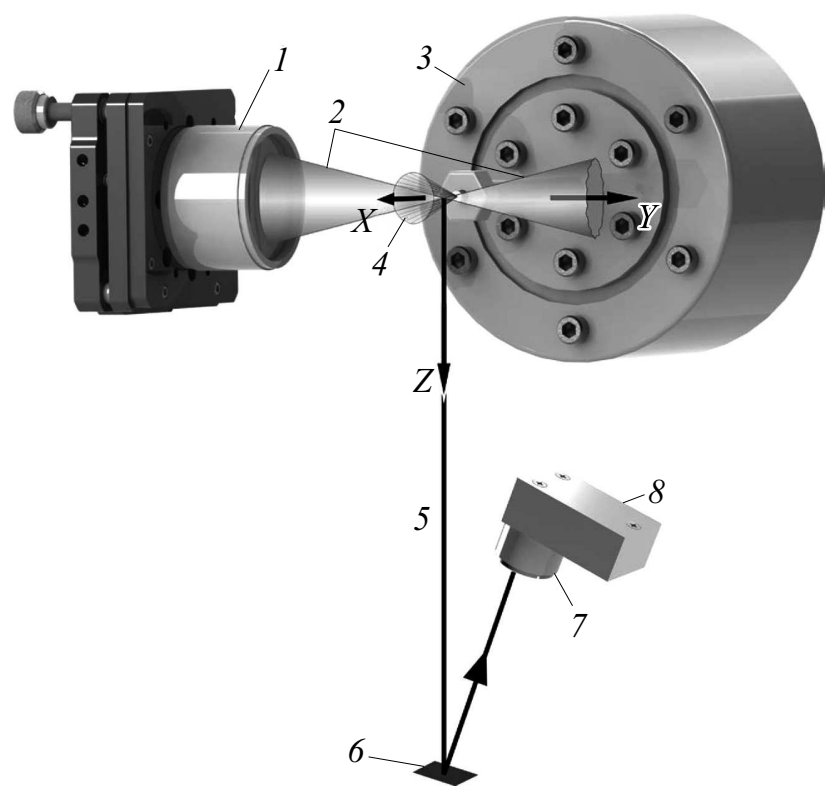

Рис. 1. Схема экспериментальной установки. $1-$ ИК-объектив, $2-$ лазерный луч, 3 - корпус генератора Хе струи, 4 - газоструйная мишень, 5 - луч наблюдения, 6 - интерференционное зеркало, 7 - держатель спектральных фильтров $\mathrm{Si} / \mathrm{Mo}, 8-$ фотодиодный датчик EUV-излучения с предусилителем в экранирующем корпусе.

через спектральный фильтр $\mathrm{Si} / \mathrm{Mo}$, подавляющий длинноволновую часть спектра, и поступало на Si-фотодиодный датчик [14]. Ось лазерного луча $(Y)$ была перпендикулярна оси струи $(X)$, а измерение интенсивности излучения плазмы проводилось в направлении, близком к третьему перпендикуляру $(Z)$.

В рамках настоящей работы были проведены два эксперимента, в ходе которых сопло перемещалось параллельно плоскости $Y Z$. Для каждого положения сопла измерение интенсивности излучения выполнялось с усреднением сигнала фотодиода по 15-20 импульсам. В первом эксперименте перемещение сопла происходило параллельно лучу наблюдения $(Z)$. Данные измерений интенсивности EUV-излучения в

8 Письма в ЖТФ, 2018, том 44, вып. 23 


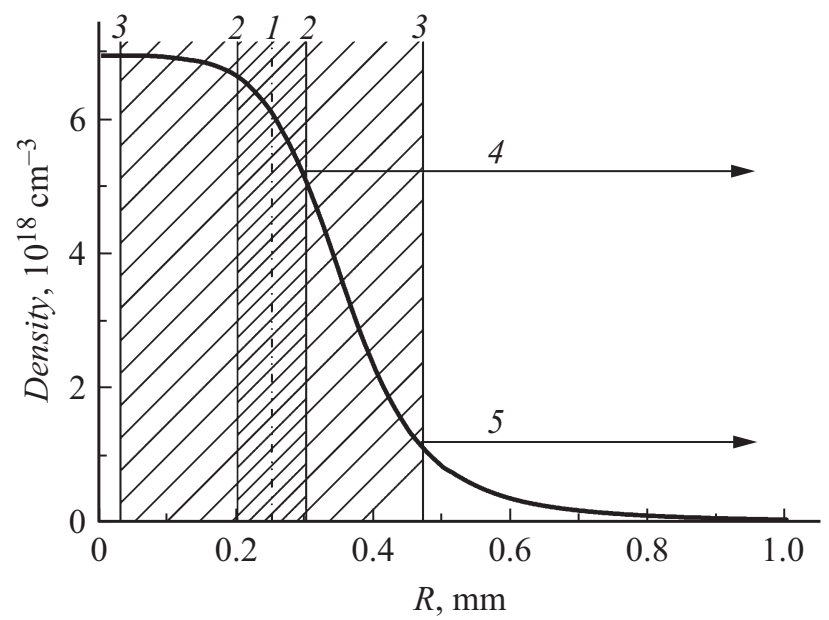

Рис. 2. Радиальное распределение плотности Хе в газоструйной мишени. 1 - положение оси лазерного луча, смещенной на $\Delta Z \approx 0.25 \mathrm{~mm}$ от оси струи в сторону наблюдателя; 2 - положение границы высокоионизованной плазмы в случае, когда лазерный луч остро сфокусирован на точку, помеченную на этом рисунке цифрой $1 ; 3$ - положение границы в случае, когда со струей пересекается расфокусированный луч; 4 и 5 - нижние пределы интегрирования и лучи, вдоль которых берется интеграл, при вычислении прозрачности периферической оболочки для случаев 2 и 3 соответственно.

зависимости от координаты $Z$ сопла показаны на рис. $3, a$. Полученный результат близок к описанному в [7-9,12]: наивысшее значение сигнала наблюдалось, когда точка фокуса была смещена от оси струи к наблюдателю примерно на 1/2 эффективного радиуса струи.

Во втором эксперименте сопло перемещалось параллельно оси лазерного луча $(Y)$, которая в соответствии с результатами первого опыта была сдвинута от оси струи к наблюдателю на половину радиуса выходного отверстия сопла, т.е. на $\Delta Z \approx 0.25 \mathrm{~mm}$. Поведение регистрируемого сигнала в зависимости от координаты $Y$ сопла оказалось совершенно неожиданным (рис. $3, b)$ : когда центр выходного отверстия сопла находился в точках $Y= \pm 1.1 \mathrm{~mm}$, т. е. когда мишень была далеко в стороне от фокуса, наблюдалось два симметрично расположенных максимума излучения, а при $Y=0$, когда фокус находился в области с

Письма в ЖТФ, 2018, том 44, вып. 23 


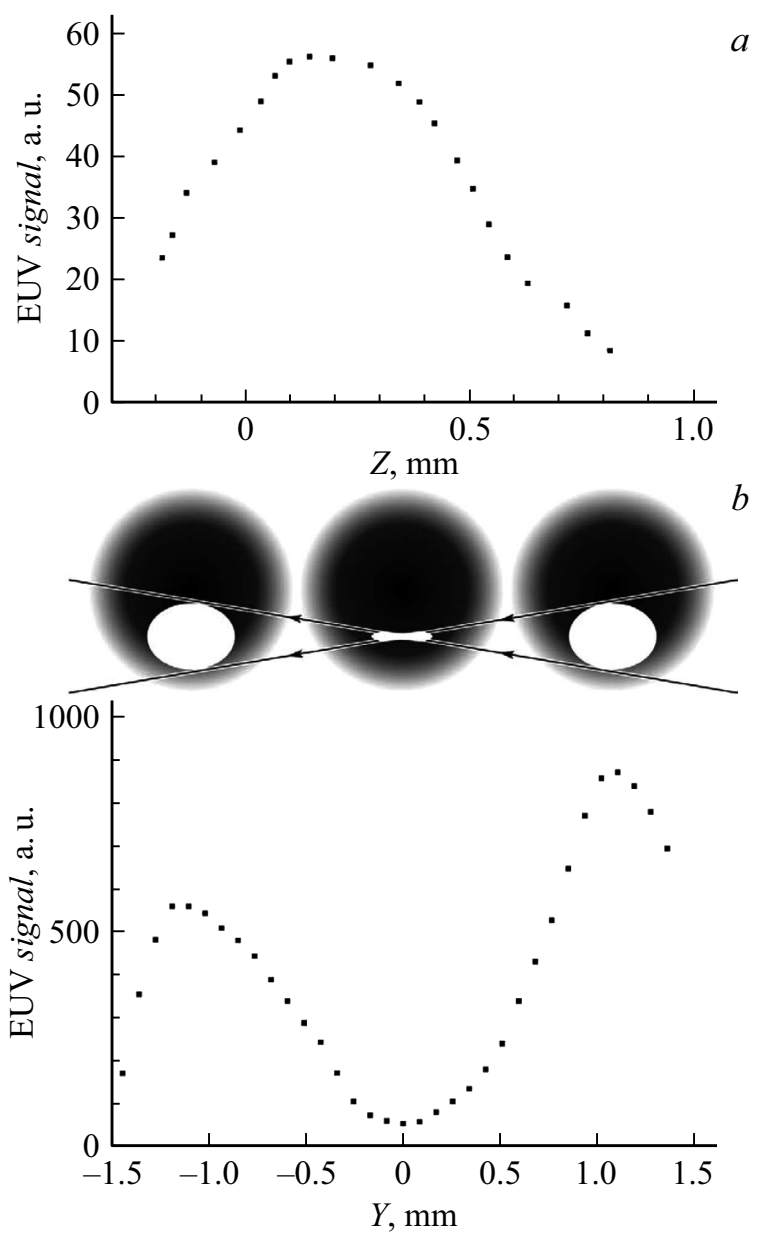

Pис. 3. $a-$ результаты опыта с перемещением сопла по отношению к фокусу вдоль луча зрения (положительные значения координаты $Z-$ смещение к наблюдателю). $b$ - опыт с перемещением вдоль оси лазерного луча $(Y)$. На вставках в верхней части белым цветом схематически изображены контуры плазмы на фоне поперечного сечения струйной мишени для трех положений оси струи вдоль лазерного луча: $Y= \pm 1.1 \mathrm{~mm}$ и $Y=0$, плотность серой заливки приближенно соответствует плотности струи, пересекающиеся прямые контуры лазерного луча, стрелки на них показывают направление от лазера.

$8^{*}$ Письма в ЖТФ, 2018, том 44, вып. 23 
наивысшей плотностью, интенсивность EUV-излучения была наинизшей (с разницей между максимумами и минимумом в 10-16 раз). Заметим, что из упомянутых выше геометрических характеристик прошедшего через объектив лазерного луча следует, что в точках $Y= \pm 1.1 \mathrm{~mm}$ диаметр луча составляет $\emptyset_{\text {beam }}=440 \mu \mathrm{m}$, в фокальной же плоскости он определяется дифракцией и по оценкам должен быть $\sim 50-100 \mu \mathrm{m}$.

Чтобы корректно описать структуру лазерной искры, созданной в фокусе луча в плотной части струи вблизи оси, обратимся к ее энергетическому балансу. Тепловая энергия выводится из плазмы посредством трех механизмов: излучательного, за счет электронной теплопроводности и гидродинамического с ионной тепловой скоростью. Были сделаны оценки характерных времен охлаждения с помощью этих механизмов для плазмы диаметром $\emptyset_{p l}=150 \mu \mathrm{m}$ с температурой $T=40 \mathrm{eV}$ (в соответствии с данными работы [5]), плотностью $n_{i}=6 \cdot 10^{18} \mathrm{~cm}^{-3}$ и ионным зарядом $\left\langle Z_{p l}\right\rangle=10$. Времена жизни энергии рассчитывались по общему правилу: $\tau_{E}=Q_{p l} / P_{l o s s}$, где $Q_{p l}=(3 / 2)(Z+1) n_{i} k_{\mathrm{B}} T V_{p l}-$ запас тепловой энергии в плазме, $k_{\mathrm{B}}-$ постоянная Больцмана, $P_{\text {loss }}=F_{\text {loss }} S_{p l}$ - мощность тепловых потерь, $F_{\text {loss }}$ - плотность потока энергии, $V_{p l}=\pi \varnothing_{p l}^{3} / 6$ и $S_{p l}=\pi \varnothing_{p l}^{2}-$ соответственно объем и площадь поверхности плазмы.

При оценке радиационных потерь энергии было выполнено моделирование плазмы с помощью online-кода FLYCHK [15], из которого следует, что интегральная по полосе $\lambda=10-15 \mathrm{~nm}$ интенсивность излучения с поверхности плоского слоя Хе плазмы с указанными выше параметрами и толщиной $150 \mu \mathrm{m}$ составляет $22-23 \%$ от излучения черного тела. Тогда для шаровой плазмы с $\varnothing_{p l}=150 \mu \mathrm{m}$ следует ожидать $P_{\text {rad }} \approx 40 \mathrm{MW}$, что совпадает с поглощаемой плазмой мощностью лазерного излучения в наших опытах (в соответствии с [16] поглощение $\left.P_{a b s} / P_{l a s} \approx 42 \%\right)$, т. е. вся поступившая в плазму энергия должна высвечиваться в виде радиационных потерь: $P_{r a d} \approx P_{a b s}-$ типичный результат для плазмы из многоэлектронных атомов. Тогда радиационное время жизни энергии $\tau_{E, \mathrm{rad}} \approx 2.7 \cdot 10^{-11} \mathrm{~s}$. Для электронной теплопроводности $\tau_{E, e-d i f f}=Q_{p l} /\left[S_{p l} \kappa_{e} \operatorname{grad}\left(k_{\mathrm{B}} T\right)\right] \approx R_{p l}^{2} /\left(3 D_{e}\right) \approx 1.2 \cdot 10^{-9} \mathrm{~s}$. Здесь $\kappa_{e}$ - электронная теплопроводность, $D_{e}=\lambda_{e i} v_{t h, e}-$ диффузионный коэффициент для электронов, $\lambda_{e i}$ и $v_{t h, e}-$ длина свободного пробега и тепловая скорость электронов, $R_{p l}=\varnothing_{p l} / 2-$ радиус плазмы. Обусловленное гидродинамическими потерями время жизни энергии 
$\tau_{E, h y d r o}=R_{p l} /\left(3 v_{t h, i}\right)=2.6 \cdot 10^{-9} \mathrm{~s}$, где $v_{t h, i} \approx 10^{6} \mathrm{~cm} / \mathrm{s}-$ тепловая скорость ионов Хе.

Итак, охлаждение плазмы практически полностью осуществляется радиационным механизмом, характерное время которого почти на три порядка меньше длительности лазерного импульса. Ясно, что такая сильно излучающая плазма будет существовать только там и тогда, где и когда существует внешний источник нагревающей ее энергии - интенсивное лазерное излучение (совпадение осциллографических форм лазерного импульса и EUV-сигнала подтверждает это заключение), т. е. лишь в пределах каустической поверхности вокруг точки фокуса. Это плазменное тело с диаметром $\leqslant 100 \mu$ m находится внутри мишени с размером $\Delta_{0.5}=0.7 \mathrm{~mm}$ и, стало быть, окружено холодным газообразным Хе, который частично ионизуется испускаемыми плазмой фотонами. Их энергии в соответствии с полосой излучения $\lambda \approx 10-15 \mathrm{~nm}$ лежат в диапазоне $h v=80-125 \mathrm{eV}$, поэтому ионный заряд ограничен сверху: $Z_{\max } \leqslant 6-8$, так как для производства ионов с более высоким $Z$ энергии излучаемых фотонов недостаточны. Если заряд ионов удалось бы увеличить $(\langle Z\rangle \geqslant 7)$, периферическая оболочка стала бы прозрачной для излучения с $\lambda \approx 13.5 \mathrm{~nm}$.

По-видимому, именно такая дополнительная ионизация и происходит при освещении струи лучом с $\varnothing_{\text {beam }}=440 \mu \mathrm{m}$. Теперь вся плотная часть мишени попадает под облучение лазером, его энергия поглощается свободными электронами, плазма нагревается, и в ней происходит процесс ионизации электронным ударом. Радиус границы высокоионизованной плазмы увеличивается от 50 до $220 \mu \mathrm{m}$ (рис. 2), а поглощение в периферической оболочке соответственно уменьшается. Если же продолжить удаление сопла от фокуса $(|Y|>1.1 \mathrm{~mm})$, интенсивность лазерного излучения падает настолько, что становится недостаточной для пробоя и нагрева плазмы. В подтверждение гипотезы были выполнены расчеты прохождения излучения с $\lambda=13.5 \mathrm{~nm}$ сквозь периферию

$$
\frac{I_{t r}}{I_{0}}=\exp \left(-\sigma_{\text {photo }} \int_{\Delta Z+R_{p l}}^{R_{j e t}} n_{i}(R) d R\right),
$$

где $\sigma_{\text {photo }}-$ сечение фотоионизации, $R_{p l}$ и $R_{\text {jet }}-$ соответственно радиусы плазмы и струи, а функция $n_{i}(R)$ приведена на рис. 2. Они показывают, что при изменении $R_{p l}$ от 50 до $220 \mu \mathrm{m}$ прозрачность

Письма в ЖТФ, 2018, том 44, вып. 23 
периферии в зависимости от предположения о ее ионном составе для нейтрального Хе увеличивается от $I_{t r} / I_{0}=0.23$ до $I_{t r} / I_{0}=0.71$, т.е. в 3 раза, а, например, для $\langle Z\rangle=5$ - от $I_{t r} / I_{0}=0.032$ до $I_{t r} / I_{0}=0.45$, т.е. в 14 раз (сечение фотоионизации из основного состояния ионов с различным $Z$ вычислялось по классической формуле Крамерса [17]: $\sigma_{\text {photo }}=2.815 \cdot 10^{29} Z^{4} / v^{3} \mathrm{~cm}^{2}$, где $v=2.2 \cdot 10^{16} \mathrm{~Hz}$ - частота ионизирующего излучения). С этим диапазоном хорошо согласуется наблюдаемое в эксперименте увеличение выхода EUV-излучения при переходе к освещению мишени широким лазерным лучом.

Таким образом, экспериментально обнаружен метод „просветления“ поглощающей периферической оболочки в Хе лазерной плазме, позволяющий существенно поднять выход излучения на длинах волн, представляющих интерес для EUV-литографии.

Работа выполнена при частичной поддержке РФФИ (проект 18-08-00716).

\section{Список литературы}

[1] Chkhalo N.I., Salashchenko N.N. // AIP Adv. 2013. V. 3. P. 082130.

[2] Chkhalo N.I., Gusev S.A., Nechay A.N., Pariev D.E., Polkovnikov V.N., Salashchenko N.N., Schäfers F., Sertsu M.G., Sokolov A., Svechnikov M.V., Tatarsky D.A. // Opt. Lett. 2017. V. 42. P. 5070-5073.

[3] Stamm U., Gäbel K. // EUV sources for lithography / Ed. V. Bakshi. Bel-lingham, WA, USA: SPIE Press, 2006. Ch. 19.

[4] O’Sullivan G. // 2011 Int. Workshop on EUV lithography. Maui, Hawaii, 2011. P. P2; http://www.euvlitho.com/2011/P2.pdf

[5] Белик В.П., Калмыков С.Г., Можаров А.М., Петренко М.В., Сасин М.Э. // Письма в ЖТФ. 2017. Т. 43. В. 22. С. 10-17.

[6] Буторин П.С., Задиранов Ю.М., Зуев С.Ю., Калмыков С.Г., Полковников В.Н., Сасин М.Э., Чхало Н.И. // ЖТФ. 2018. Т. 88. В. 10. С. 1554-1558.

[7] Капралов В.Г., Корде Р., Левашов В.Е., Пирожков А.С., Рагозин Е.Н. // Квантовая электроника. 2002. Т. 32. № 2. С. 149-154.

[8] Левашов В.Е., Медников К.Н., Пирожков А.С., Рагозин Е.Н. // Квантовая электроника. 2006. Т. 36. № 6. С. 549-552.

[9] Bobashev S.V., Domracheva I.V., Petrenko M.V., Stepanova Z.A. // Proc. of SPIE. 2007. V. 6614. P. 66140D.

[10] de Bruijn R., Koshelev K.N., Bijkerk F. // J. Phys. D: Appl. Phys. 2003. V. 36. P. L88-L91. 
[11] de Bruijn R., Koshelev K.N., Zakharov S.V., Novikov V.G., Bijkerk F. // Phys. Plasmas. 2005. V. 12. P. 042701.

[12] Гарбарук А.В., Демидов Д.А., Калмыков С.Г., Сасин М.Э. // ЖТФ. 2011. T. 81. B. 6. C. 20-29.

[13] Garbaruk A.V., Gritskevich M.S., Kalmykov S.G., Mozharov A.M., Sasin M.E. // J. Phys. D: Appl. Phys. 2017. V. 50. P. 025201.

[14] Забродский В.В., Белик В.П., Аруев П.Н., Бер Б.Я., Бобашев С.В., Петренко М.В., Суханов В.Л. // Письма в ЖТФ. 2012. Т. 38. В. 17. С. 69-77.

[15] Chung H.-K., Chen M.H., Morgan W.L., Ralchenko Y., Lee R.W. // High Energy Density Phys. 2005. V. 1. P. 3-12.

[16] Борисевичус Д.А., Забродский В.В., Калмыков С.Г., Сасин М.Э., Сейсян Р.П. // Письма в ЖТФ. 2017. Т. 43. В. 1. С. 53-60.

[17] Методы исследования плазмы. Спектроскопия, лазеры, зонды / Под ред. В. Лохте-Хольтгревена. М.: Мир, 1971. С. 31. 